\title{
Impact of direct stenting on clinical outcomes for small vessel coronary artery disease in patients undergoing primary percutaneous coronary intervention for ST-elevation myocardial infarction
}

\author{
Kahraman Cosansu ${ }^{1}$, Cagin Mustafa Ureyen², Mehmet Bulent Vatan ${ }^{1}$, Mustafa Tarik Agac ${ }^{1}$, Harun Kilic ${ }^{1}$, \\ Ramazan Akdemir ${ }^{1}$ \\ ${ }^{1}$ Department of Cardiology, Sakarya University Education and Research Hospital, Sakarya, Turkey \\ ${ }^{2}$ Department of Cardiology, Antalya Education and Research Hospital, Antalya, Turkey
}

Adv Interv Cardiol 2019; 15, 4 (58): 404-411

DOI: https://doi.org/10.5114/aic.2019.90214

\begin{abstract}
Introduction: Direct stenting (DS) is associated with improved markers of reperfusion during primary percutaneous coronary intervention (PPCI) for ST-elevation myocardial infarction (STEMI). However, data evaluating its impact in small vessel coronary artery disease (CAD) are lacking.

Aim: To compare DS and conventional stenting (CS) for small vessel CAD on clinical outcomes of patients with STEMI undergoing $\mathrm{PPCl}$.

Material and methods: A cohort of 616 STEMI patients treated with DS (202 patients) or CS (414 patients) in small vessel $(\leq 2.75 \mathrm{~mm}$ ) lesions was retrospectively analyzed. The primary endpoint was to compare the occurrence of major adverse cardiac events (MACE) between groups during 2-year follow-up. The secondary end points included in-hospital target lesion revascularization (TLR) and in-hospital death.

Results: The primary end-point, MACEs, occurred in 9.2\% in the DS group and $12.3 \%$ in the CS group $(p>0.05)$. The rates of TLR, myocardial infarction (MI) and target vessel revascularization (TVR) were not significantly different between groups $(p>0.05)$. The stent thrombosis (ST) rate was significantly lower in the DS group (1.0\% vs. $4.2 \%, p=0.04)$ at 2 years. However, DS was not found to be an independent predictor of ST in multivariate analysis. There were no significant differences in in-hospital rates of death and TLR. The DS compared to CS resulted in greater rates of postprocedural TIMI grade 3 flow, and lower risk of edge dissection. The procedure time, radiation exposure and contrast administration were found to be significantly lower in the DS group.

Conclusions: In selected patients with STEMI undergoing PPCI for small vessel CAD, DS is not only safe and feasible but also reduces ST rates, contrast load, and procedural and radiation exposure time.
\end{abstract}

Key words: direct stenting, ST-elevation myocardial infarction, primary percutaneous coronary intervention, conventional stenting, small vessel coronary artery.

Su m m a ry

The impact of direct stenting on small vessel coronary culprit lesions in patients with ST-segment elevation myocardial infarction has not been investigated yet. The present study clearly emphasizes that direct stenting in selected lesions appears to be a safe and successful procedure, providing lower stent thrombosis and procedural complication rates. The procedural and radiation exposure time, and contrast load were also lower in the DS group.

\section{Introduction}

The primary therapeutic strategy in patients with acute ST-elevation myocardial infarction (STEMI) is per- cutaneous coronary intervention (PCI) with stent implantation [1]. The conventional stenting (CS) technique requires routine pre-dilatation with the balloon catheter

\section{Corresponding author:}

Kahraman Cosansu MD, Department of Cardiology, Sakarya University Education and Research Hospital, Sakarya, Turkey,

e-mail: kahraman141@gmail.com

Received: 5.03.2019, accepted: 1.07.2019. 
to ensure easy passage of the stent and to increase the full expansion of stent. With the innovation in stent and delivery system design, direct stenting without balloon pre-dilatation has become a feasible strategy in many catheterization laboratories [2]. Direct stenting (DS) without lesion pre-dilatation is employed in $30-40 \%$ of $\mathrm{PCls}$ and has been compared to stenting with pre-dilatation in observational studies as well as randomized trials. DS without balloon pre-dilatation is thought to provide beneficial effects by reducing distal embolization and thereby microvascular obstruction [3]. Prospective studies as well as meta-analyses demonstrate $22-50 \%$ reductions in restenosis, target lesion revascularization (TLR), myocardial infarction (MI) and death associated with DS compared to CS [4-6]. Pre-dilation may induce intimal dissection necessitating multiple or altogether longer stents, increasing the risk of restenosis [7]. DS also offers significant reductions in procedure time, radiation exposure, contrast administration, and adjunctive material, use and also cost reduction was achieved by direct stenting [8]. However, a number of disadvantages have been suggested for DS, including failure to cross the lesion, incomplete stent deployment, an increase in guide trauma, undersizing the stent, and poor visualization, which may result in errors in stent positioning [9]. It also might increase procedural risks and may lead to suboptimal clinical results.

\section{Aim}

There are no previous studies comparing DS with a conventional approach for small vessel coronary artery disease (CAD) for clinical outcomes of patients with ST-elevation myocardial infarction (STEMI). The aim of the study was to assess in-hospital and long-term clinical outcomes of STEMI patients with small vessel CAD treated with direct stenting as compared with those treated with stenting after pre-dilatation.

\section{Material and methods}

\section{Study design and population}

This was a retrospective, observational, single-center study. The current study included 616 consecutive patients undergoing a primary $\mathrm{PCl}$ with stenting for small vessels $(\leq 2.75 \mathrm{~mm})$ in Sakarya University Education and Research Hospital, Sakarya, Turkey from January 2013 to January 2017. The patients were divided into two groups, treated with DS ( $n=202,32.8 \%)$ or CS $(n=414,67.2 \%)$. Small vessel CAD was considered a need for implantation of stents $\leq 2.75 \mathrm{~mm}$ (diameter of the reference vessel and diameter of the implanted stent: $\leq 2.75 \mathrm{~mm}$ ). All patients $>18$ years of age presenting with STEMI within $12 \mathrm{~h}$ of symptom onset or between 12 and $24 \mathrm{~h}$ if they had persistent symptoms with evidence of ongoing ischemia were included in the study. DS was the primary modality of treatment in all patients wherever possi- ble. All patients where DS was done formed the direct stenting group (DS group), and the rest were included in the CS group. Patients in the CS group underwent balloon pre-dilatation prior to stenting. The exclusion criteria were a concomitant large diameter $\mathrm{PCl}$ in the same coronary artery, left main coronary artery lesions, contraindications to inhibit platelet function with aspirin and clopidogrel, cardiogenic shock, a $\mathrm{PCl}$ consisting of in-stent restenosis (ISR) for the culprit lesion, life expectancy $<12$ months and pregnancy. Lesions that were heavily calcified or associated with excessive proximal tortuosity were also excluded. An additional exclusion criterion was a lack of relevant patient or procedural related data.

This study complied with the Declaration of Helsinki, and it was approved by the independent medical ethics committee of Sakarya University Education and Research Hospital.

\section{Study protocol}

During primary $\mathrm{PCl}$, those patients who had thrombolysis in myocardial infarction (TIMI) flow of $\geq 1$ at initial injection or after wire placement underwent DS. Stenting was performed after pre-dilatation in patients whose vessel was still completely occluded after insertion of the wire. Coronary stenting was considered angiographically successful if residual stenosis of $<30 \%$ and coronary thrombolysis in myocardial infarction grade flow 3 were obtained at the end of the procedure. During the procedure, an intra-arterial bolus of unfractionated heparin was given at a dose of $80 \mathrm{U} / \mathrm{kg}$. After the intervention, all patients received aspirin indefinitely, clopidogrel, prasugrel or ticagrelor for at least 12 months and other cardiac medications according to ACC/AHA guidelines [10]. Angiographic findings such as vessel dimensions, pre- and post-procedural stenoses and lesion length were determined by visual estimation using the guiding catheter as a reference object for calibration. The angiographic characteristics were also further analyzed by an independent interventional cardiologist not involved in the procedure and checked for inter-observer agreement.

\section{Data collection}

Baseline demographics, clinical characteristics and procedural data were collected retrospectively. Thrombus burden, calcification status and postprocedural TIMI flow grade were evaluated by two experienced interventional cardiologists. The contrast volume, procedural time and fluoroscopy time data were obtained from the records of the coronary angiography laboratory. The in-hospital and 2-year follow-up information on clinical outcomes (e.g. in- hospital death, recurrent MIs, TLR, target vessel revascularization (TVR) and definite ST) were collected from electronic medical records, a registry database or phone calls, which asked about relevant end-point clini- 
cal events. Routine or control angiography during the follow-up without a clinical indication was not undertaken. However, event-driven coronary angiographies after the initial $\mathrm{PCl}$ were performed within the 2-year follow-up period.

\section{Study endpoints and definitions}

The primary end-point of the study was the composite of major adverse cardiac events (MACEs), which were defined as TLR, TVR, MI or definite ST during the follow-up period. The secondary end-points included in-hospital TLR and in-hospital death. TVR was defined as any clinically driven $\mathrm{PCl}$ or bypass grafting of the target vessel. TLR was defined as any clinically driven repeat $\mathrm{PCl}$ or bypass grafting of the treated lesion, including the placement of an in-stent or in-segment $5 \mathrm{~mm}$ proximal or distal to the initial stent edges. An MI was defined according to current guidelines [11]. Definite ST was defined based on the criteria of the Academic Research Consortium [12]. Total ischemic time was defined as the time from the onset of chest pain to the first balloon inflation during primary $\mathrm{PCl}$. Angiographic thrombus burden was graded using TIMI thrombus classification, and it was classified as low thrombus burden (grades 1, 2 and 3) and high thrombus burden (grades 4 and 5) [13]. In this study, no-reflow and edge dissection were defined as procedural complications.

\section{Statistical analysis}

For the statistical analysis, SPSS version 16.0 for Windows (SPSS Inc., Chicago, IL) was used. Continuous data were expressed as mean \pm standard deviation, and the categorical data were expressed as percentages. The normal distribution of the data was assessed by the Kolmogorov-Smirnov test. Comparisons between groups were performed using the $\chi^{2}$ or Fisher's exact test for qualitative variables, as appropriate. The independent $t$-test was used for normally distributed continuous variables, and the Mann-Whitney $U$ test was conducted for non-normally distributed continuous variables, as appropriate. The baseline characteristics and clinical outcomes of the patients treated with direct stenting versus those treated with pre-dilatation prior to stenting were compared. Kaplan-Meier analysis was used to calculate the time to the clinical end-point, and the log-rank test was applied to compare between-group differences. To determine the impact of the DS strategy on 2-year ST, multivariate logistic analysis was performed. Clinical, procedural and angiographic criteria were entered into a univariate model with 2-year ST as the dependent variable. Variables reaching significance, or borderline significance, on univariate analysis $(p \leq 0.1)$ were subsequently incorporated into a multivariate model. Independent variables are presented as odds ratios with $95 \%$ confidence intervals. The Cox model included age, gender, multivessel disease, stent length, stent diameter, target vessel, lesion location, complication, postdilatation, prior $\mathrm{PCl}$, prior $\mathrm{MI}$, prior CABG, diabetes mellitus (DM), hypertension ( $\mathrm{HT}$ ), hyperlipidemia, smoking, renal failure, thrombus burden, left ventricular ejection fraction (LVEF), and DS. For multivariate analysis, the following covariates were considered for entry into the multivariate models (only those with $p$-values $<0.10$ were retained via stepwise regression): DS, gender, prior $\mathrm{PCl}$, prior $\mathrm{MI}$, and stent diameter. A $p$-value $<0.05$ was considered statistically significant in all tests.

\section{Results}

\section{Characteristics of patients}

The baseline demographics and baseline clinical characteristics of groups are shown in Table I. The two groups were comparable in terms of demographics and clinical characteristics and all values were similar between the two groups $(p>0.05)$. However, significantly lower baseline $\operatorname{LVEF}(p=0.009)$ and higher age $(p=0.019)$ were found in the CS group. The total ischemic time was found similar in both groups.

\section{Characteristics of the lesions, and PCI procedures}

The lesional and procedural characteristics are summarized in Table II. There was no difference in terms of culprit artery and lesion location between groups. In both groups, the lesions were located mostly in the middle part of the vessel and the culprit artery was mostly the right coronary artery $(R C A)$. Moreover, the stents were significantly longer $(p=0.001)$ and stent diameter was lower $(p=0.050)$ in the CS group. The incidence of procedural complications were significantly higher in the CS group as compared with the DS group $(p=0.01)$. The DS compared to CS resulted in greater rates of postprocedural TIMI grade 3 flow $(p=0.048)$ and lower risk of edge dissection $(p=0.038)$. However, there was no difference between the two groups with regards to thrombus burden, calcification, or rate of aspiration thrombectomy. DS when compared with the CS technique significantly decreased both procedure time $(43.5 \pm 12.6 \mathrm{~min}$ vs. 47.7 $\pm 15.2 \mathrm{~min}, p<0.001)$ and fluoroscopy time $(9.7 \pm 4.4 \mathrm{~min}$ vs. $11.7 \pm 5.7 \mathrm{~min}, p<0.001)$. Moreover, contrast volume used in the DS group was significantly lower than the CS group $(128.8 \pm 52.6 \mathrm{ml}$ vs. $151.3 \pm 83.7 \mathrm{ml}, p<0.001)$.

\section{Clinical outcomes}

At the 2-year follow-up, 52 (25.7\%) patients in the DS group and 98 (23.7\%) patients in the CS group needed to undergo angiographic evaluations. The outcomes of the patients during the follow-up period are summarized in Table III. At the 2-year follow-up, the primary composite end-point, MACEs, occurred in 9.2.\% of the DS group and 
Table I. Baseline demographics and clinical characteristics

\begin{tabular}{lccc} 
Variables & DS $(\boldsymbol{n}=\mathbf{2 0 2})$ & CS $(\boldsymbol{n}=\mathbf{4 1 4})$ & $\boldsymbol{P}$-value \\
\hline Male, $n(\%)$ & $142(70.3)$ & $300(72.5)$ & 0.505 \\
\hline Age, mean \pm SD [years] & $63.0 \pm 11.9$ & $65.4 \pm 12.1$ & 0.019 \\
\hline Smoker, $n(\%)$ & $102(50.5)$ & $191(46.1)$ & 0.309 \\
\hline Ejection fraction, mean \pm SD (\%) & $47.7 \pm 9.2$ & $45.5 \pm 10.7$ & 0.009 \\
\hline Diabetes mellitus, $n(\%)$ & $81(40.1)$ & $168(40.7)$ & 0.891 \\
\hline Hypertension, $n(\%)$ & $93(46.0)$ & $188(45.5)$ & 0.903 \\
\hline Hyperlipidemia, $n(\%)$ & $36(17.8)$ & $93(22.5)$ & 0.179 \\
\hline Prior MI, $n(\%)$ & $19(9.4)$ & $40(9.7)$ & 0.919 \\
\hline Prior PCI, $n(\%)$ & $20(9.9)$ & $34(8.2)$ & 0.487 \\
\hline Prior CABG, $n(\%)$ & $3(1.5)$ & $12(2.9)$ & 0.286 \\
\hline Renal insufficiency, $n(\%)$ & $25(12.4)$ & $64(15.5)$ & 0.302 \\
\hline TI time, mean \pm SD [min] & $116.5 \pm 40.6$ & $113.8 \pm 38.9$ & 0.435
\end{tabular}

Data presented as mean \pm standard deviation or number (\%). DS - direct stenting, CS - conventional stenting, MI - myocardial infarction, PCI - percutaneous coronary intervention, $C A B G$ - coronary artery bypass graft, TI time - total ischemic time.

Table II. Lesions and procedural characteristics

\begin{tabular}{|c|c|c|c|}
\hline Parameter & DS $(n=202)$ & $\operatorname{CS}(n=414)$ & $P$-value \\
\hline \multicolumn{4}{|l|}{ Culprit artery: } \\
\hline Left anterior descending & $60(29.8)$ & $139(33.5)$ & 0.165 \\
\hline Left circumflex & $15(7.4)$ & $69(16.7)$ & \\
\hline Right coronary & $113(55.9)$ & $158(38.2)$ & \\
\hline Other & $14(6.9)$ & $48(11.6)$ & \\
\hline Procedural complication: & $15(7.5)$ & $61(14.7)$ & 0.010 \\
\hline Edge dissection & $7(3.0)$ & $27(6.5)$ & 0.038 \\
\hline No reflow & $8(4.5)$ & $34(8.2)$ & 0.059 \\
\hline \multicolumn{4}{|l|}{ Procedural characteristics: } \\
\hline Post-dilation & $27(13.4)$ & $48(11.6)$ & 0.528 \\
\hline Aspiration thrombectomy & $12(5.9)$ & $23(5.6)$ & 0.847 \\
\hline Stent length, mean \pm SD [mm] & $18.67 \pm 5.93$ & $20.85 \pm 6.17$ & 0.001 \\
\hline Stent diameter, mean \pm SD $[\mathrm{mm}]$ & $2.48 \pm 0.10$ & $2.46 \pm 0.14$ & 0.050 \\
\hline Procedure time, mean $\pm \mathrm{SD}[\mathrm{min}]$ & $43.5 \pm 12.6$ & $47.7 \pm 15.2$ & $<0.001$ \\
\hline Fluoroscopy time, mean \pm SD [min] & $9.7 \pm 4.4$ & $11.7 \pm 5.7$ & $<0.001$ \\
\hline Contrast volume, mean \pm SD [ml] & $128.8 \pm 52.6$ & $151.3 \pm 83.7$ & $<0.001$ \\
\hline Postprocedural TIMI flow III, $n$ (\%) & $190(94.1)$ & $371(89.6)$ & 0.048 \\
\hline \multicolumn{4}{|l|}{ Lesion location: } \\
\hline Proximal & $63(31.1)$ & $153(37.0)$ & 0.586 \\
\hline Mid & $111(55.0)$ & $193(46.6)$ & \\
\hline Distal & $28(13.9)$ & $68(16.4)$ & \\
\hline Additional stent, $n$ (\%) & $42(20.8)$ & $89(21.5)$ & 0.841 \\
\hline Multivessel disease, $n(\%)$ & $27(13.4)$ & $39(9.4)$ & 0.137 \\
\hline Calcific lesion, $n(\%)$ & $27(13.4)$ & $65(15.7)$ & 0.446 \\
\hline \multicolumn{4}{|l|}{ Thrombus burden: } \\
\hline Low thrombus burden & $146(72.3)$ & $302(72.9)$ & 0.847 \\
\hline High thrombus burden & $56(27.7)$ & $112(27.1)$ & \\
\hline
\end{tabular}

Data are presented as mean \pm standard deviation or number (\%). DS - direct stenting, CS - conventional stenting, TIMI - thrombolysis in myocardial infarction. 
Table III. Clinical outcomes at 2 years and in-hospital

\begin{tabular}{lccc} 
Parameter & DS $(\boldsymbol{n}=\mathbf{2 0 2})$ & CS $(\boldsymbol{n}=4 \mathbf{4 1 4})$ & $\boldsymbol{P}$-value \\
\hline MI & $12(6.2)$ & $38(10.0)$ & 0.130 \\
\hline TLR & $6(3.1)$ & $25(6.6)$ & 0.079 \\
\hline TVR & $11(5.7)$ & $30(7.9)$ & 0.336 \\
\hline ST & $2(1.0)$ & $16(4.2)$ & 0.040 \\
\hline MACE & $18(9.2)$ & $47(12.3)$ & 0.265 \\
\hline In-hospital death & $9(4.5)$ & $34(8.2)$ & 0.086 \\
\hline In-hospital TLR & $3(1.5)$ & $6(1.4)$ & 0.972
\end{tabular}

Data are $n$ (\%). DS - direct stenting, CS - conventional stenting, MI - myocardial infarction, TLR - target lesion revascularization, TVR - target vessel revascularization, ST - stent thrombosis, MACE - major adverse cardiovascular events.

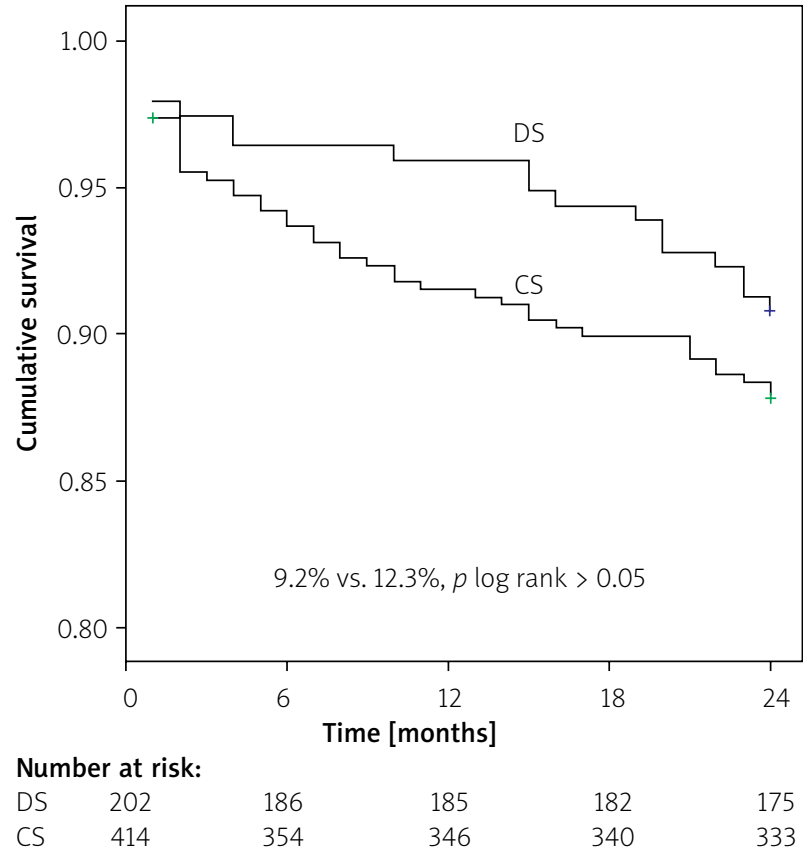

Figure 1. Kaplan-Meier curve estimate of freedom from major adverse cardiac events (MACE) at 24-month follow-up

DS - direct stenting, CS - conventional stenting.

$12.3 \%$ of the CS group $(p>0.05)$. There was also no significant difference in TVR and $M I$ rates between the two groups after 2 years $(p>0.05)$. Moreover, there was no significant difference between groups in TLR rates during hospitalization $(p>0.05)$. Although TLR and in-hospital death tended to be lower in the DS group, the differences between groups were not significant $(p=0.07$ and $p=0.08$, respectively). However, the ST rate was significantly higher in the CS group as compared with the DS group ( $p=0.04)$.

Kaplan-Meier survival analysis revealed no difference in event-free MACE ratio between the patients treated with DS and CS (9.2\% vs. $12.3 \%$; $p$ log rank > 0.05) (Fig-

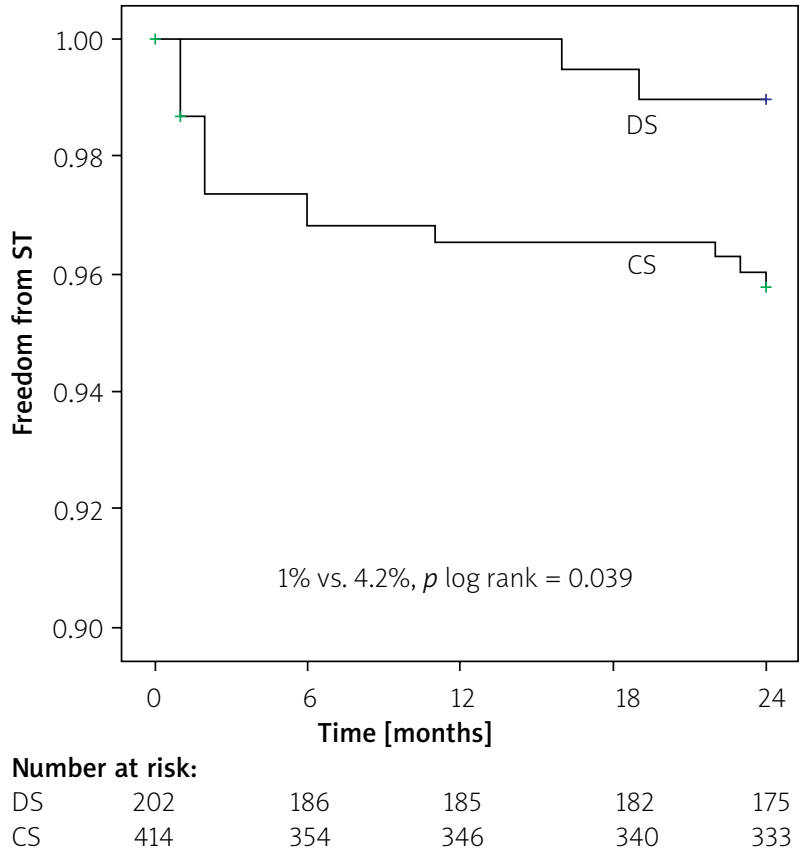

Figure 2. Kaplan-Meier curve estimate of freedom from stent thrombosis (ST) at 24 month follow-up DS - direct stenting, CS - conventional stenting.

ure 1). The time-to-event curves reflected a higher incidence of ST in the CS group for small-vessel culprit lesions (4.2\% vs. $1.0 \%$; $p$ log rank = 0.039) (Figure 2 ).

DS was found to be a predictor of ST at 2 years (hazard ratio $(\mathrm{HR})=0.07,95 \%$ confidence interval $(\mathrm{Cl})$ : $0.01-0.66, p=0.021$ ) in univariate Cox analysis. However, when adjusted for confounding factors, DS was not an independent predictor of ST at 2 years in multivariate Cox analysis ( $\mathrm{HR}=0.24 ; 95 \% \mathrm{Cl}: 0.05-1.04 ; p=0.058)$.

\section{Discussion}

This is the first study to include STEMI patients with small vessel coronary artery disease who were treated 
with DS or CS and report their long-term outcomes. The main findings of the current study are as follows: First, the rate of MACEs, the primary end-point, was similar in the direct stenting group as compared with that in the patients treated with conventional stenting. Second, there were no statistically significant difference in TLR, TVR, MI, in-hospital death and in-hospital TLR. However, conventional stenting was associated with a significantly higher stent thrombosis rate at 2-year follow-up and also higher procedural complication. DS was not found to be an independent predictor of ST at 2 years in multivariate analysis. Moreover, DS was associated with a shorter procedure and fluoroscopy time, as well as lower contrast volume and postprocedural TIMI III flow.

DS is defined as the technique of coronary stent implantation without predilatation by balloon. To perform direct stenting, it is mandatory to visualize the length of the culprit lesion and the diameter of the vessel. However, in a STEMI patient, TIMI flow is most often $\leq 1$ before wire placement [14]. During primary $\mathrm{PCl}$, patients who had TIMI flow of $\geq 2$ at initial injection or after wire placement are suitable for direct stenting. The direct stenting technique has a number of theoretical advantages. Prospective studies and meta-analyses have consistently demonstrated significant benefit of DS in terms of safety, procedural outcomes, MACE rate and mortality [15]. Superior clinical outcomes associated with DS may be driven by reduced wall damage and inflammatory response from balloon predilation [16], and fewer intimal dissections [17]. Balloon predilation before stenting is associated with higher risk of distal embolization and microvascular occlusion, which is associated with more reperfusion failure and lower probability of final TIMI 3 flow $[3,18]$. In our study, a relatively high incidence of procedural complications and lower TIMI III flow rate were observed in the conventional stent group versus the direct stent group, compatible with previous results. However, underestimation of true vessel size, failure to cross the lesion, non-dilatable lesions, inadequate stent expansion and late stent malapposition were possible limitations of DS [19].

Routine use of manual thrombus aspiration in primary $\mathrm{PCl}$ resulted in improved myocardial reperfusion and reduced 1-year cardiac mortality in the TAPAS study [20]. However, the efficacy of routine thrombus aspiration could not be confirmed in either the TASTE [21] or the TOTAL [22] trial, prompting international guidelines to advise its use in selected patients only [23]. In a recent study, Mahmoud et al. reported that clinical outcomes and myocardial reperfusion measures did not differ significantly between DS and CS and there was no interaction with thrombus aspiration [24]. In this study, we used thrombus aspiration only in selected patients, and thrombus aspiration was used at a similar rate in both groups.
The results of studies investigating the usefulness of the DS strategy in terms of clinical outcomes are contradictory. In recent years, several meta-analyses comparing DS and CS have been published. However, these studies have not been performed in STEMI patients or in small vessel coronary artery disease. Therefore, the outcome rates in our study are important in terms of being the first data in this patient group. Magalhaes et al. reported significantly lower MACE and TLR rates following DS as compared with CS in a meta-analysis of elective procedures [15]. In another meta-analysis, myocardial infarction was significantly reduced with direct stenting compared with conventional stenting, but the target-vessel revascularization rate was similar between groups [6]. Moreover, Alak et al. found a significant reduction in the risk of in-hospital cardiovascular death, but no significant differences were observed in myocardial infarction or target lesion revascularization [25]. Different results were found in each of these three metaanalyses, which might have resulted from studies with different methodologies in different patient groups. They included many old treatment elements, including antiplatelet therapy with ticlopidine and implantation of mostly bare metal stents.

In the literature, there are few studies comparing DS and CS in terms of clinical outcomes in STEMI patients. Kalayci et al. found that DS in primary $\mathrm{PCl}$ was associated with better postprocedural angiographic results and long-term survival; however, the DS group had similar in-hospital and long-term mortality to matched patients in the CS group [26]. In another study, direct stenting compared to conventional stenting was associated with a significantly lower rate of all-cause death and stroke, but there were non-significant differences in target lesion revascularization, myocardial infarction, stent thrombosis and major bleeding at 1-year follow-up [27]. McCormick et al. reported that patients receiving DS had reduced mortality at 30-day and 1-year follow-up [28]. In the EUROTRANSFER registry, direct compared with conventional stenting resulted in significantly higher rates of postprocedural TIMI grade 3 flow, lower risk of no-reflow and significant reduction in 1-year mortality [29]. In the present study, the rate of edge dissection was lower and the rate of TIMI III flow was higher in the DS group. However, there was no difference in terms of no-reflow between groups. In-hospital death and TLR at 2 years were also lower in the DS group, but it did not reach significance. The rate of stent thrombosis is higher in patients with small vessel CAD as compared with those with non-small vessel CAD in the literature [30]. In the previous studies ST rates were found to be similar in DS and CS groups in non-small vessel CAD. In the current study, although the stent thrombosis rate was higher in the CS group in STEMI patients with small-vessel CAD, DS was not found to be an independent predictor of ST at 2 years in multivariate analysis. Increased stent throm- 
bosis in the CS group may be associated with the use of longer and smaller diameter stents in this group. In addition, the suboptimal procedural result (TIMI flow grade $<3$ ) is a predictor of ST, and in our study the TIMI III flow rate was lower in the CS group.

\section{Limitations}

Several limitations of the present study should be acknowledged. First, the present trial was a single centre pilot study representing the experience of only one hospital and the results can only be hypothesis-generating. Second, only about $25 \%$ of our patients received follow-up coronary angiography according to clinical indications, and the potential bias related to the incomplete angiographic follow-up might have had a substantial impact on the analytic results. Third, a bias cannot be excluded as to which patients seemed suitable for direct stent implantation.

\section{Conclusions}

As in our study, there is no other study comparing DS and CS in terms of clinical outcomes in small-vessel CAD patients with STEMI. Direct stenting without predilation in selected lesions seems to be a safe and successful procedure that provides a lower stent thrombosis and procedural complication rate and potential advantages as savings in procedural time, contrast load, and shortened radiation exposure time. However, larger and randomized studies with longer follow-up are mandatory before routine clinical use is recommended.

\section{Conflict of interest}

The authors declare no conflict of interest.

\section{References}

1. Windecker S, Bax JJ, Myat A, et al. Future treatment strategies in ST-segment elevation myocardial infarction. Lancet 2013; 382: 644-57.

2. Ormiston JA, Webster MWI, Ruygrok PN, et al. A randomized study of direct coronary stent delivery compared with stenting after predilatation: the NIR future trial. Catheter Cardiovasc Interv 2000; 50: 377-81.

3. Barbato E, Marco J, Wijns W. Direct stenting. Eur Heart J 2003; 24: 394-403.

4. Ly HQ, Kirtane AJ, Buros J, et al. Angiographic and clinical outcomes associated with direct versus conventional stenting among patients treated with fibrinolytic therapy for ST-elevation acute myocardial infarction. Am J Cardiol 2005; 95: 383-6.

5. Burzotta F, Trani C, Prati F, et al. Comparison of outcomes (early and six-month) of direct stenting with conventional stenting (a meta-analysis of ten randomized trials). Am J Cardiol 2003; 91: 790-6.

6. Piscione F, Piccolo R, Cassese $\mathrm{S}$, et al. Is direct stenting superior to stenting with predilation in patients treated with percutaneous coronary intervention? Results from a meta-analysis of 24 randomized controlled trials. Heart 2010; 96: 588-94.
7. Foley DP, Pieper M, Wijns W, et al. The influence of stent length on clinical and angiographic outcome in patients undergoing elective stenting for native coronary artery lesions; final results of the Magic 5L study. Eur Heart J 2001; 22: 1585-93.

8. Caluk J, Osmanovic E, Barakovic F, et al. Direct coronary stenting in reducing radiation and radiocontrast consumption. Radiol Oncol 2010; 44: 153-7.

9. Dawkins KD, Chevalier B, Suttorp MJ, et al. Effectiveness of 'direct' stenting without balloon predilatation (from the Multilink Tetra Randomised European Direct Stent Study [TRENDS]). Am J Cardiol 2006; 97: 316-21.

10. O'Gara PT, Kushner FG, Ascheim DD, et al. 2013 ACCF/AHA guideline for the management of ST-elevation myocardial infarction: executive summary: a report of the American College of Cardiology Foundation/American Heart Association Task Force on Practice Guidelines. Circulation 2013; 127: 529-55.

11. Thygesen K, Alpert JS, Jaffe AS, et al. Third universal definition of myocardial infarction. J Am Coll Cardiol 2012; 60: 1581-98.

12. Cutlip DE, Windecker S, Mehran R, et al. Academic Research Consortium. Clinical end points in coronary stent trials: a case for standardized definitions. Circulation 2007; 115: 2344-51.

13. Topaz O, Topaz A, Owen K. Thrombus grading for coronary interventions: the role of contemporary classifications. Interv Cardiol 2011; 3: 705-12.

14. Bailleul C, Puymirat E, Aissaoui N, et al. Factors associated with infarct-related artery patency before primary percutaneous coronary intervention for ST-Elevation myocardial infarction (from the FAST-MI 2010 Registry). Am J Cardiol 2016; 117: 17-21.

15. Magalhaes MA, Minha S, Lhermusier T, et al. Does direct stenting with drug-eluting stents improve outcome? A meta-analysis of 10,900 patients. Catheter Cardiovasc Interv 2017; 90: 213-22.

16. Ormiston JA, Mahmud E, Turco MA, et al. Direct stenting with the TAXUS Liberte drug-eluting stent: results from the Taxus Atlas Direct Stent Study. JACC Cardiovasc Interv 2008; 1: 150-60.

17. Wilson SH, Berger PB, Mathew V, et al. Immediate and late outcomes after direct stent implantation without balloon predilation. J Am Coll Cardiol 2000; 35: 937-43.

18. Azzalini L, Millan X, Ly HQ, et al. Direct stenting versus pre-dilation in ST-elevation myocardial infarction: a systematic review and meta-analysis. J Interv Cardiol 2015; 28: 119-31.

19. Seto A, Kern M. Direct stenting for STEMI: does it really make a difference? Catheter Cardiovasc Interv 2014; 84: 932-3.

20. Vlaar PJ, Svilaas T, van der Horst IC, et al. Cardiac death and reinfarction after 1 year in the Thrombus Aspiration during Percutaneous Coronary Intervention in Acute myocardial infarction Study (TAPAS): a 1-year follow-up study. Lancet 2008; 371: 1915-20.

21. Frobert O, Lagerqvist B, Olivecrona GK, et al.; TASTE Trial. Thrombus aspiration during ST-segment elevation myocardial infarction. N Engl J Med 2013; 369: 1587-97.

22. Jolly SS, Cairns JA, Yusuf S, et al.; TOTAL Investigators. Randomized trial of primary PCl with or without routine manual thrombectomy. N Engl J Med 2015; 372: 1389-98.

23. Ibanez B, James S, Agewall S, et al.; ESC Scientific Document Group. 2017 ESC Guidelines for the management of acute myocardial infarction in patients presenting with ST-segment elevation: the Task Force for the management of acute myocardial infarction in patients presenting with ST-segment elevation of the European Society of Cardiology (ESC). Eur Heart J 2018; 39: 119-77. 
24. Mahmoud KD, Jolly SS, James S, et al. Clinical impact of direct stenting and interaction with thrombus aspiration in patients with ST-segment elevation myocardial infarction undergoing percutaneous coronary intervention: Thrombectomy Trialists Collaboration. Eur Heart J 2018; 39: 2472-9.

25. Alak A, Lugomirski P, Aleksova N, et al. A meta-analysis of randomized controlled trials of conventional stenting versus direct stenting in patients with acute myocardial infarction. J Invasive Cardiol 2015; 27: 405-9.

26. Kalayci A, Oduncu V, Karabay CY, et al. Outcomes of direct stenting in patients with ST-elevated myocardial infarction. Herz 2018; 43: 447-54.

27. Mockel M, Vollert J, Lansky AJ, et al. Comparison of direct stenting with conventional stent implantation in acute myocardial infarction. Am J Cardiol 2011; 108: 1697-703.

28. McCormick LM, Brown AJ, Ring LS, et al. Direct stenting is an independent predictor of improved survival in patients undergoing primary percutaneous coronary intervention for ST elevation myocardial infarction. Eur Heart J Acute Cardiovasc Care 2014; 3: 340-6.

29. Dziewierz A, Siudak Z, Rakowski T, et al. Impact of direct stenting on outcome of patients with ST-elevation myocardial infarction transferred for primary percutaneous coronary intervention (from the EUROTRANSFER registry). Catheter Cardiovasc Interv 2014; 84: 925-31.

30. Gao R, Abizaid A, Banning A, et al. One-year outcome of small-vessel disease treated with sirolimus-eluting stents: a subgroup analysis of the e-SELECT registry. I Interv Cardiol 2013; 26: 163-72. 